CLINICAL ALERT

\title{
Listerial brainstem encephalitis - treatable, but easily missed
}

\author{
P Fredericks, M Britz, R Eastman, J A Carr, K J Bateman
}

Pearl Fredericks and Jonathan Carr are affiliated to the Division of Neurology, Department of Internal Medicine, Tygerberg Hospital and Faculty of Medicine and Health Sciences, Stellenbosch University, Tygerberg, Cape Town, South Africa. Marcell Britz is a specialist neurologist in private practice in Port Elizabeth, Eastern Cape, South Africa. Roland Eastman and Kathleen Bateman are affiliated to the Division of Neurology, Department of Medicine, Groote Schuur Hospital and Faculty of Health Sciences, University of Cape Town, South Africa.

Corresponding author: K Bateman (kathleen.bateman@uct.ac.za)

Listerial brainstem encephalitis (LBE) is an uncommon form of listerial central nervous system infection that progresses rapidly and is invariably fatal unless detected and treated early. We report on six adult patients with LBE, of whom five were managed or co-managed by our unit during the period January - June 2012. All presented with a short prodromal illness followed by a combination of brainstem signs, including multiple cranial nerve palsies with emphasis on the lower cranial nerves, ataxia, motor and sensory long-tract signs, a depressed level of consciousness and apnoea. In two cases the diagnosis was delayed with adverse outcomes. LBE may be difficult to diagnose: clinicians may not be aware of this condition, the brainstem location may not be recognised readily, general markers of inflammation such as the erythrocyte sedimentation rate, C-reactive protein level or white cell count may be normal, and the cerebrospinal fluid is typically normal or there are only mild and nonspecific findings. Serological tests are unreliable, and diagnosis is achieved through blood cultures, magnetic resonance imaging and clinical recognition.

S Afr Med J 2015;105(1):17-20. DOI:10.7196/SAMJ.8700

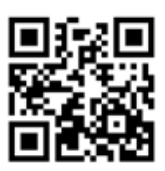

Listeria monocytogenes is a very common intracellular Gram-positive coccobacillus occurring in the soil, and humans may be affected via the oral route from contaminated food. The organism has a remarkable resistance to eradication during food processing, has unique virulence factors, ${ }^{[1,2]}$ and is an important cause of food-borne epidemic disease with high associated mortality. ${ }^{[3]}$ Following ingestion, infection occurs across the intestinal epithelium and spreads to the liver, where it becomes established within the cytosol of macrophages. Macrophages may then traffic infection via the bloodstream to the meninges and fetoplacental unit. ${ }^{[1]}$ The disease usually presents as a febrile diarrhoeal illness, but may also cause septicaemia, meningitis and chorioamnionitis. Listerial meningitis may be the third most common cause of acute community-acquired bacterial meningitis. ${ }^{[2]}$

Listerial brainstem meningitis (LBE), however, is an uncommon, sporadically occurring form of listerial infection that progresses rapidly and is invariably fatal unless treated early. ${ }^{[3-5]}$ Cases are often undetected. ${ }^{[5]}$ It is characterised by a prodromal febrile illness followed within 4 - 10 days by the abrupt onset of cranial nerve deficits associated with encephalopathy, hemiparesis, hemisensory loss and/ or cerebellar signs. ${ }^{[3-5]}$ Unlike the other forms of central nervous system (CNS) listeriosis, $\mathrm{LBE}$ is striking in respect of the stepwise development of brainstem signs in the absence of meningism. This presentation is probably accounted for by a novel route of infection that exploits retrograde axonal transport to the brainstem along the cranial nerves that supply the oropharynx. ${ }^{[2,6]}$

\section{Methods}

We report on six patients with LBE, of whom five were managed or co-managed by the neurology unit at Tygerberg Hospital, Western Cape, South Africa, during the period January - June 2012. Definite cases were defined as cases with a compatible clinical syndrome (prodrome followed by stepwise neurological deficits due to brainstem disease) and microbiological demonstration of L. monocytogenes in blood, cerebrospinal fluid (CSF) or brain tissue. Probable cases were defined as cases with a compatible clinical syndrome with ancillary tests (brain imaging and CSF analysis) supporting the diagnosis and evidence of improvement after commencement of antilisterial therapy.

The Stellenbosch University Human Research Ethics Committee approved the reporting of clinical material (S13/10/223).

\section{Case report}

The index case (patient 1) was a 59-year-old woman who presented with a 1-week flu-like illness followed by ataxia and sensory loss involving the face. Thereafter she developed a deteriorating level of consciousness and died within 3 weeks.

At onset she complained of fever, dizziness and unremitting frontal headache, and she was treated with telithromycin followed by moxifloxacin for suspected sinusitis. One week later, she developed hoarseness, numbness of the left cheek and ataxia of the left arm. A chest radiograph was normal. Magnetic resonance imaging (MRI) of the brain showed signal abnormalities in the pons and left middle cerebellar peduncle on fluid-attenuated inversion recovery (FLAIR) sequences, with subtle contrast enhancement on T1-weighted images. Intravenous (IV) methylprednisolone was given for 3 days for a presumptive diagnosis of acute disseminated encephalomyelitis (ADEM), during which her condition remained stable and she was apyrexial. However, a day later, she developed a fever and became comatose, and was found to have bilateral papilloedema. CSF protein and glucose levels were normal, and there were 4 polymorphonuclear cells per high-power field on microscopy. A CSF Gram stain was negative, and neither the CSF nor initial blood cultures yielded growth after 5 days of incubation. Repeat MRI of the brain showed extension of signal abnormalities into both cerebellar peduncles, the cerebellum 


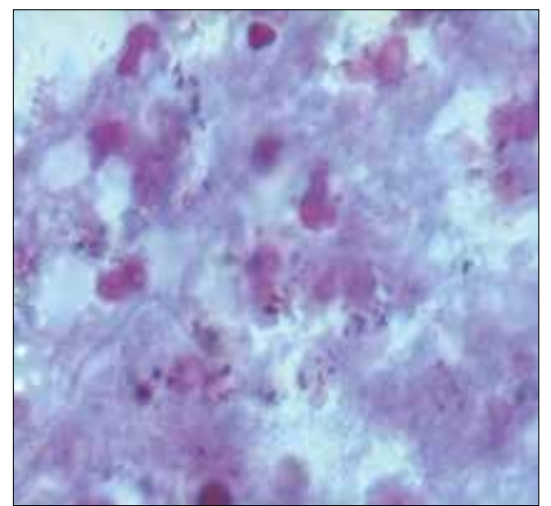

Fig. 1. Oil-immersion photomicrograph at $\times 1000$ magnification shows Gram-positive short intracellular rods.

and rostrally into the basal ganglia. There was no clinical response to treatment with IV corticosteroids and acyclovir. Subsequent MRI brain imaging revealed progression of disease to involve the upper cervical cord, brainstem, cerebellum, bilateral basal ganglia and rostral left cerebral hemisphere. A repeat CSF sample was acellular with normal chemistry and microscopy. The patient died after 17 days of illness. Blood cultures taken on day 15 as well as CSF cultures taken on day 17 cultured L. monocytogenes, and at autopsy L. monocytogenes microabscesses were confirmed (Fig. 1).

\section{Discussion}

We report two definite (patients 1 and 4) and four probable cases of LBE. The clinical data and ancillary tests are summarised in Tables 1 and 2, respectively. LBE typically begins with a prodrome of up to 16 days (median 4 days), ${ }^{[3,4]}$ with isolated cases reported to have prodromes of more than a month. ${ }^{[3]}$ In our series, the median time before the onset of neurological signs was 5.5 days. Headache $(73 \%)$, fever $(85 \%)$ and nausea or vomiting $(64 \%)$ are the most commonly reported early features, ${ }^{[3,4]}$ but these were absent in a third of our patients. Mental state at admission is often preserved (59\%). ${ }^{[3]}$ The prodrome is followed by the onset of focal signs of lower brainstem and cerebellar involvement. Patients frequently present with asymmetrical cranial nerve dysfunction (VII, VI, IX, X and $\mathrm{V}$, in order of descending frequency). ${ }^{[3-5]}$ Untreated patients deteriorate over days with new cranial nerve deficits and the emergence of 'crossed' or bilateral longtract signs (hemiparesis more often than hemisensory loss), cerebellar signs and/ or encephalopathy. At maximal evolution, most patients have combined cranial nerve deficits and long-tract signs (81\%), and more than half have cerebellar signs. ${ }^{[3-5]}$ Cranial

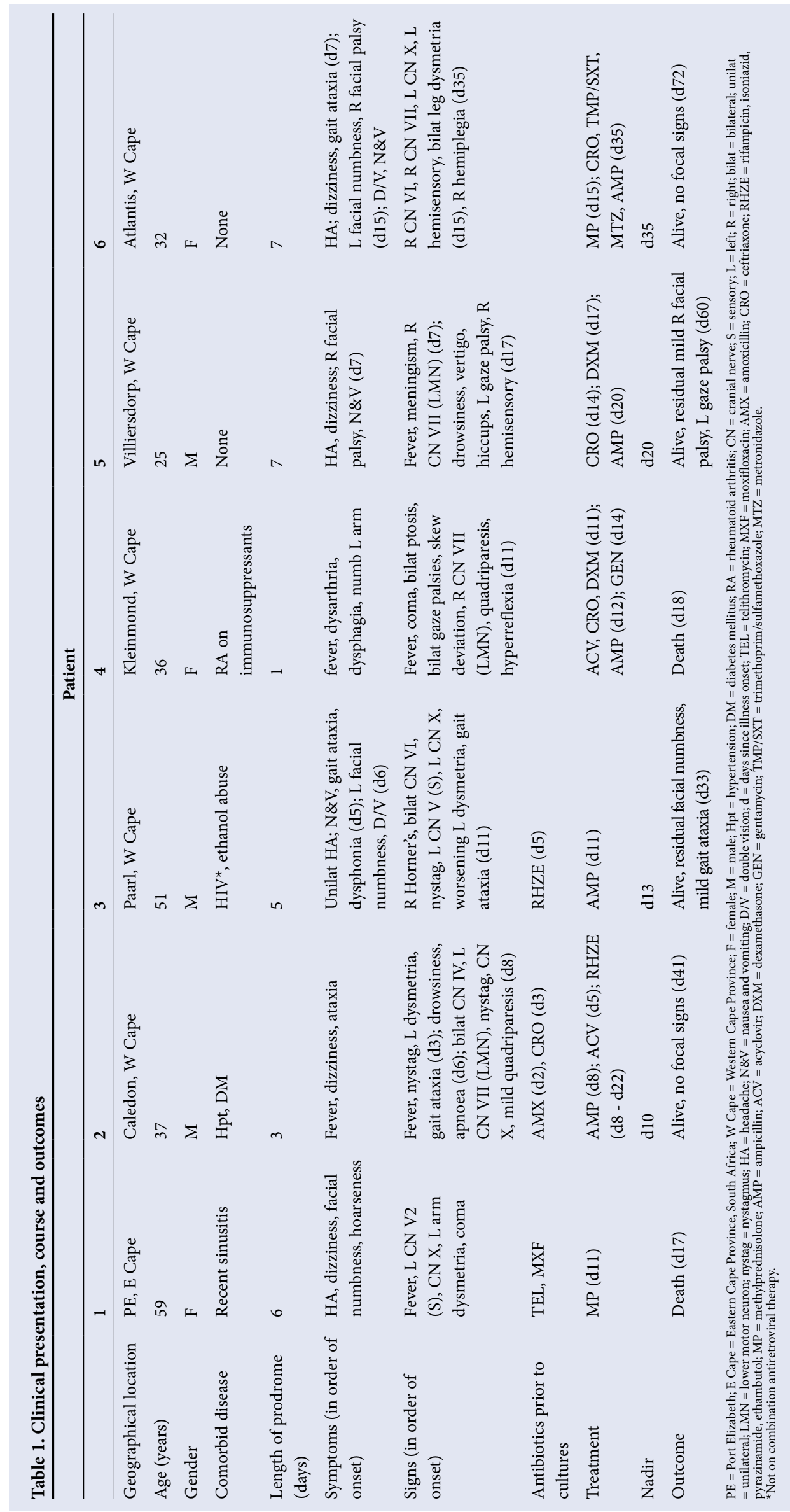


Table 2. Laboratory, pathology and radiological investigations

\begin{tabular}{|c|c|c|c|c|c|c|}
\hline & \multicolumn{6}{|c|}{ Patient } \\
\hline & 1 & 2 & 3 & 4 & 5 & 6 \\
\hline \multicolumn{7}{|l|}{ Inflammatory markers } \\
\hline WCC & 13 & 13.07 & 6.52 & 12.17 & 15.31 & 9.57 \\
\hline CRP & ND & 97 & 86 & 85 & ND & ND \\
\hline \multicolumn{7}{|l|}{ CSF } \\
\hline Cells (PMN, Ly, E) & $4,0,0$ & $11,484,5$ & $66,21,6$ & $12,68,9$ & $4,375,0$ & $0,60,0$ \\
\hline Protein $(g / L)$ & 0.3 & 0.8 & 0.69 & 0.5 & 0.59 & 0.63 \\
\hline Glucose $(\mathrm{mmol} / \mathrm{L})$ & 6.3 & 6 & 3.2 & 2.8 & 3.3 & 3.3 \\
\hline \multicolumn{7}{|l|}{$\begin{array}{l}\text { L. monocytogenes } \\
\text { culture }\end{array}$} \\
\hline Blood & Pos (d15) & $\operatorname{Neg}(\times 2)$ & $\mathrm{Neg}(\times 2)$ & $\operatorname{Pos}(\times 2)$ & $\operatorname{Neg}(\times 2)$ & $\operatorname{Neg}(\times 2)$ \\
\hline CSF & Pos (d17) & $\mathrm{Neg}$ & $\mathrm{Neg}$ & ND & $\mathrm{Neg}$ & $\mathrm{Neg}$ \\
\hline Brain biopsy & Pos & ND & ND & ND & ND & ND \\
\hline CT & ND & $\mathrm{N}$ & $\begin{array}{l}\text { Well-defined } \\
\text { ring lesions } \\
\text { dorsal Po, } \\
\text { L MCP }\end{array}$ & $\begin{array}{l}\text { Poorly defined } \\
\text { lesions } \\
\text { R Po, R MCP; } \\
\text { incomplete } \\
\text { ring } \\
\text { enhancement }\end{array}$ & $\mathrm{N}$ & $\mathrm{N}$ \\
\hline \multicolumn{7}{|l|}{ MRI } \\
\hline $\begin{array}{l}\text { Distribution of high signal on } \\
\text { T2WI \& FLAIR sequences }\end{array}$ & $\begin{array}{l}\text { L Po, MCP, } \\
\text { and } \mathrm{Cb}\end{array}$ & $\begin{array}{l}\text { L cerebral ped, } \\
\text { L MCP, L Cb }\end{array}$ & ND & $\begin{array}{l}\text { R cerebral ped, } \\
\text { R MCP, Me, } \\
\text { upper cord }\end{array}$ & $\begin{array}{l}\mathrm{R} \text { cerebral ped, } \\
\mathrm{R} \text { MCP, dorsal } \\
\text { Po, Me, upper } \\
\text { cord }\end{array}$ & $\begin{array}{l}\text { Mb, dorsal Po, } \\
\text { Cb vermis, } \\
\text { Me; after } 9 \mathrm{~d} \\
\text { interval: } \\
\text { new lesions } \\
\mathrm{L} \text { thalamic, } \\
\mathrm{L} \text { parietal }\end{array}$ \\
\hline $\begin{array}{l}\text { Enhancement pattern on T1WI } \\
\text { pre-/post gadolinium }\end{array}$ & $\begin{array}{l}\text { Subtle, } \\
\text { incomplete } \\
\text { ring dorsal Po }\end{array}$ & Complete ring & ND & $\begin{array}{l}\text { Incomplete } \\
\text { ring }\end{array}$ & $\begin{array}{l}\text { Patchy, } \\
\text { nodular }\end{array}$ & $\begin{array}{l}\text { Patchy, } \\
\text { nodular; } \\
\text { complete ring }\end{array}$ \\
\hline $\mathrm{CN}$ enhancement present & $\mathrm{N}$ & $\mathrm{Y}$ & $\mathrm{Y}$ & $\mathrm{Y}$ & $\mathrm{N}$ & $\mathrm{N}$ \\
\hline Radiological differential reported & $\begin{array}{l}\text { Demyelination } \\
\text { (BS/ADEM), } \\
\text { glioma }\end{array}$ & Abscess & $\begin{array}{l}\text { Tuberculoma, } \\
\text { listerial } \\
\text { abscess }\end{array}$ & $\begin{array}{l}\text { Demyelination } \\
\text { (ADEM) }\end{array}$ & $\begin{array}{l}\text { Demyelination } \\
\text { (ADEM) }\end{array}$ & $\begin{array}{l}\text { Abscess, } \\
\text { demyelination }\end{array}$ \\
\hline
\end{tabular}

nerve involvement is seen in all patients at the nadir of the illness. Frequently, noncontiguous cranial nerve nuclei will be affected during the course of the illness; for instance, patient 6 had left trigeminal sensory involvement, right abducens palsy and lower motor neuron facial weakness, as well as a left palatal palsy (cranial nerve $\mathrm{X}$ ), indicating multifocal brainstem involvement. Respiratory failure due to involvement of the medulla may occur early and necessitate ventilatory support. ${ }^{[3,5]} \mathrm{A}$ reduced level of consciousness was present in four of the six patients.

The differential diagnosis of brainstem encephalitis varies depending on population and geographical region. ${ }^{[7]}$ Broadly, this presentation may be due to demyelination (e.g. multiple sclerosis, ADEM) or autoimmune/inflammatory (e.g. Behçet's disease, paraneoplastic), infectious and very rarely neoplastic diseases (e.g. lymphoma) ${ }^{[7,8]}$ Infectious causes in adults include viruses, such as entero- and herpesviruses. Surprisingly, however, a bacterial infection such as listeria is one of the most common infectious causes of brainstem encephalitis. In an SA setting, tuberculosis and progressive multifocal leucoencephalopathy due to JC virus infection are also important considerations in the setting of progressive brainstem disease.
With CNS invasion, listeriosis may target the meninges resulting in the typical clinical picture of acute/subacute bacterial meningitis, ${ }^{[2]}$ but unlike other bacteria may also target the brain parenchyma and specifically the rhombencephalon (pons, medulla, cerebellum). In humans, brainstem infection occurs in up to $11-24 \%$ of patients with CNS listeriosis. ${ }^{[3,5]}$ In LBE, it appears that food-borne bacteria invade the brainstem by retrograde axonal migration along the oropharyngeal cranial nerves. ${ }^{[2]}$ A postmortem study of nine human cases of LBE revealed brainstem microabscesses that were prominently distributed within the nuclei, tracts and intraparenchymal 
portions of the cranial nerves innervating the oropharynx (V, VII, IX, X and XII)..$^{[9]}$ Injection of listerial bacteria into the facial nerves of mice was followed 5 - 10 days later by ipsilateral CNS deficits that were prevented by section of the nerve proximal to the inoculation site. ${ }^{[10]}$ Similarly, injection of listerial bacteria into the sciatic nerves of mice resulted in a flaccid paraparesis that was prevented by sectioning the sciatic nerve proximal to the inoculation site. ${ }^{[10]}$ Actindependent locomotion of $L$. monocytogenes along microtubules has been demonstrated in other eukaryote cells and remains the most plausible explanation for bacterial propagation along axons. ${ }^{[1]}$

Factors predisposing to LBE in humans have not been identified. Unlike listerial meningitis, where two-thirds of patients are immunosuppressed by medication, advanced age or comorbid disease, LBE has been reported to occur mainly in immunocompetent adults. ${ }^{[3]}$ Of our patients, three had comorbid conditions such as immunosuppressant therapy, diabetes, alcoholism and HIV infection.

Of importance, CSF abnormalities in LBE are not typical of bacterial infections of the CNS, and one in five patients may have normal CSF on initial sampling. ${ }^{[3]} \mathrm{A}$ relatively normal CSF glucose level, moderate lymphocytic pleocytosis and a moderately increased CSF protein level may mistakenly be attributed to viral or mycobacterial infection, or, as in one of our patients, to inflammatory demyelination. ${ }^{[3-5]}$ The CSF was normal in one of our patients, and patient 3 had a neutrophil-predominant mild pleocytosis.

In a review of patients with LBE by Armstrong and Fung, ${ }^{[3]}$ CSF Gram stains were positive in only $10 \%$ of cases, with CSF cultures positive in $33-41 \%$ and blood cultures in $61 \%$. Positive cultures were

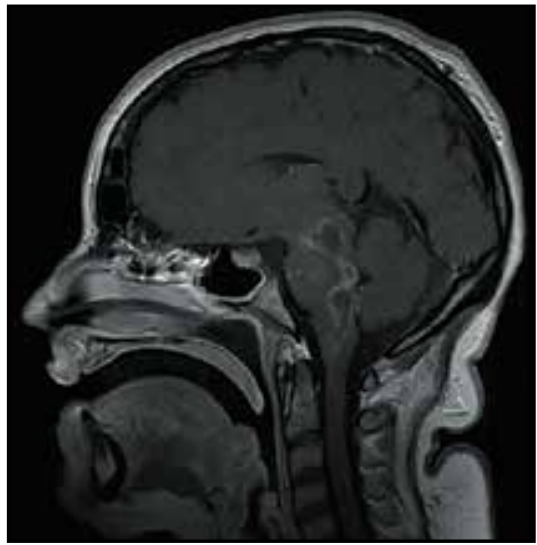

Fig. 2. Sagittal MRI brain scan (T1WI post gadolinium) of patient 4 showing rim-enhancing lesions in the brainstem. (MRI = magnetic resonance imaging.) obtained in two of our six patients (two on blood, one on CSF).

MRI is the radiological investigation of choice in the diagnosis of brainstem disease. In our series, findings on MRI were abnormal in all patients studied (five scanned, see example in Fig. 2), and showed T2 signal change in the brainstem, cerebellar peduncles and cerebellum associated with variable enhancement on contrasted T1-weighted images. Computed tomography (CT) is less sensitive than MRI in LBE; ${ }^{[3-5]}$ two of five patients in this study had abnormal findings. Striking cranial nerve enhancement may be seen on MRI ${ }^{[12]}$ or, less often, on CT, and was present in three of our six patients (two MRI, one CT). Radiological appearances are nonspecific, however, and in a region of high tuberculosis prevalence listerial brainstem abscesses may be radiologically indistinguishable from tuberculomas. In the absence of microbiological or histopathological confirmation, empiric treatment for both L. monocytogenes and Mycobacterium tuberculosis may have to be considered. Rapid radiological and clinical improvement would favour listeriosis over tuberculosis as the likely cause.

Supratentorial abscess formation may occur in conjunction with LBE and was present in patients 1 and 6 in our series. ${ }^{[3,13]}$ Following the prodrome and initial neurological deficit, patient 6 received high-dose corticosteroids for a presumed diagnosis of ADEM, after which disease progression appeared stable until abrupt deterioration on day 35 . MRI showed left thalamic and parietal lobe abscess formation. A biopsy specimen of brain tissue for culture, obtained 5 days after starting intravenous ampicillin, was negative. The patient made a remarkable recovery.

High-dose ampicillin is the drug of choice. The recommended treatment is ampicillin 2 g IV 4-hourly for 21 days, ${ }^{[14]}$ although some authors suggest longer treatment periods and the addition of an aminoglycoside ${ }^{[15]}$ Gentamicin acts synergistically with ampicillin and may be added in patients with immune impairment. ${ }^{[3]}$ Co-trimoxazole is an acceptable alternative in penicillinallergic patients. ${ }^{[14]}$ It is important to note that third-generation cephalosporins, commonly recommended as first-line therapy for bacterial meningitis of unknown cause, have no activity against Listeria species.

Untreated patients invariably die, ${ }^{[3-5]}$ usually within $5-18$ days after symptom onset. ${ }^{[3]}$ Treated survival rates of $49-64 \%$ have been reported. ${ }^{[3,4]}$ Early treatment with ampicillin is associated with improved survival $(76 \%)$ compared with delayed treatment or initial treatment with inappropriate antibiotics such as cephalosporins. ${ }^{[3]}$ Neurological dysfunction improves within $2-7$ days of drug initiation. ${ }^{[4]}$ In our series, no patient suffered disabling sequelae.

Small epidemics of listeriosis from contaminated food sources are frequently reported in countries where the infection is notifiable, and the source of the contaminated food has often been identified. Given the high fatality rate of listeriosis, contaminated food is an important public health hazard. In SA, however, the disease is not notifiable at present. It is of interest that, as three patients in this series presented within 6 months of each other and came from geographically closely related areas, the infection may have been caused by a common contaminated food source.

\section{References}

1. Freitag NE, Port GC, Miner MD. Listeria monocytogenes from saprophyte to intracellular pathogen. Nat Rev Microbiol 2009;7(9):623-628. [http://dx.doi.org/10.1038/nrmicro2171]

2. Disson O, Lecuit M. Targeting of the central nervous system by Disson O, Lecuit M. Targeting of the central nervous system by
Listeria monocytogenes. Virulence 2012;3(2):213-221. [http:// Listeria monocytogenes. Virulence
dx.doi.org/ $10.4161 \% 2$ Fviru. 19586

dx.doi.org/ $10.4161 \% 2$ Fviru. 19586$]$
Armstrong RW, Fung PC. Brainstem encephalitis (rhombencephalitis) due to Listeria monocytogenes: Case report and review. Clin Infect Dis 1993;16(5):689-702. [http://dx.doi. org/10.1093/clind/16.5.689]

4. Uldry PA, Kuntzer T, Bogousslavsky J, et al. Early symptoms and outcome of Listeria monocytogenes rhombencephalitis: 14 adult cases. J Neurol 1993;240(4):235-242. [http://dx.doi.org/10.1007/ BF00818711]

5. Antal E-A, Dietrichs E, Løberg E-M, Melby KK, Maehlen J. Brain stem encephalitis in listeriosis. Scand J Infect Dis 2005;37(3):190194. [http://dx.doi.org/10.1080/00365540410020938]

6. Madarame H, Seuberlich T, Abril C, Zurbriggen A, Vandevelde 6erigen A, Vandevelde $\mathrm{M}$, Oevermann $\mathrm{A}$. The distribution of $\mathrm{E}$-cadherin expression in listeric rhombencephalitis of ruminants indicates its involvement in Listeria monocytogenes neuroinvasion. Neuropathol Appl Neurobiol 2011;37(7):753-767. [http://dx.doi. org/10.1111/j.1365-2990.2011.01183.x

7. Jubelt B, Mihai C, Li TM, Veerapaneni P. Rhombencephalitis/ brainstem encephalitis. Curr Neurol Neurosci Rep 2011;11(6):543552. [http://dx.doi.org/10.1007/s11910-011-0228-5]

8. Moragas M, Martínez-Yélamos S, Majós C, Fernández-Viladrich P, Rubio F, Arbizu T. Rhombencephalitis: A series of 97 patients. Medicine (Baltimore) 2011;90(4):256-261. [http://dx.doi. org/10.1097/MD.0b013e318224b5af]

9. Antal E-A, Løberg E-M, Dietrichs E, Maehlen J. Neuropathological findings in 9 cases of Listeria monocytogenes Neuropathological findings in 9 cases of Listeria monocytogenes
brain stem encephalitis. Brain Pathol 2005;15(3):187-191. brin stem encephalitis. Brain Pathol 2005;15(3):

10. Antal E-A, Løberg E-M, Bracht P, Melby KK, Maehlen J. Evidence for intraaxonal spread of Listeria monocytogenes from the periphery to the central nervous system. Brain Pathol 2001;11(4):432-438. [http://dx.doi.org/10.1111/j.1750-3639.2001. tb00411.x

11. Dons L, Jin Y, Kristensson K, Rottenberg ME. Axonal transport of Listeria monocytogenes and nerve-cell-induced bacterial killing. J Neurosci Res 2007;85(12):2529-2537. [http://dx.doi. org/10.1002/jnr.21256

12. Moro A, Albino PHDC, Bresciani AP, et al. Rhombencephalitis caused by Listeria monocytogenes with striking involvement of trigeminal nerve on MR imaging. Arq Neuropsiquiatr 2011:69(3):568-569. [http://dx.doi. org/10.1590/S0004-282X2011000400033]

13. Soares-Fernandes JP, Beleza P, Cerqueira JI, et al. Simultaneous supratentorial and brainstem abscesses due to Listeria supratentorial and brainstem abscesses due to Listeria
monocytogenes. J Neuroradiol 2008;35(3):173-176. [http:// monocytogenes.
dx.doi.org/10.1016/j.neurad.2007.07.009]

dx.doi.org/10.1016/j.neurad.2007.07.009]
14. Van de Beek D, Brouwer MC, Thwaites GE, Tunkel AR. 14. Van de Beek D, Brouwer MC, Thwaites GE, Tunkel AR.
Advances in treatment of bacterial meningitis. Lancet 2012;380(9854):1693-1702. [http://dx.doi.org/10.1016/S01406736(12)61186-6]

15. Mylonakis E, Hohmann EL, Calderwood SB. Central nervous system infection with Listeria monocytogenes: 33 years' experience at a general hospital and review of 776 episodes from the literature. Medicine (Baltimore) 1998;77(5):313-336. [http:// dx.doi.org/10.1097/00005792-199809000-00002] 